\title{
A Highly Efficient Rectenna Using Harmonic Rejection Capability
}

\author{
Youghwan Kim $\cdot$ Sungjoon Lim
}

\begin{abstract}
A highly efficient $2.4 \mathrm{GHz}$ rectenna is designed using a harmonic rejection bandpass filter. The rectenna is printed on Rogers Duroid 5880 substrate with $\varepsilon_{r}=2.2$ and a thickness of $1.6 \mathrm{~mm}$. The rectenna consists of a microstrip antenna and high order harmonic rejection bandpass filter, microstrip lowpass filter, and Schottky barrier diode (HSMS2820). The use of a $2^{\text {nd }}$ and $3^{\text {rd }}$ harmonic rejection microstrip bandpass filter in the rectenna results in high conversion efficiency. The proposed rectenna achieves a RF to DC conversion efficiency of $72.17 \%$ when the received $\mathrm{RF}$ power is $63.09 \mathrm{~mW}$.
\end{abstract}

Key words: Rectenna, Microstrip Antenna, Micro-Strip Bandpass Filter, Harmonic Rejection, Microstrip Lowpass Filter.

\section{Introduction}

Microwave power transmission is currently an active area of research and the rectenna is one of the most important devices for microwave power transmission. Rectennas can potentially be used in a number of different applications, ranging from ubiquitous sensors and power transmission from space to earth, to wireless computer mice and keyboards. The most important parameter of a rectenna is its RF-DC conversion efficiency. Much research has focused on determining highly efficient designs for rectennas.

In general, three methods are recognized for increasing the efficiency of the rectenna. The most important component is a rectifying diode. High conversion efficiency from RF to DC can be dominantly achieved by good rectifying diodes. A rectenna requires a small amount of energy, which means that the rectenna must use low forward voltage (threshold voltage) diodes. However, the selection of the most suitable diode to make a highly efficient rectenna system cannot be handled by RF designers. Design of a high gain antenna is another way to produce a highly efficient rectenna because it can receive and deliver RF power to the rectifying circuit. Various antennas, including dual-band [1], circular sector [2], and broadband [3] antennas have been introduced in previous literature for use in rectennas. The third approach is to block the re-radiation of signal. The received RF signal from the antenna is converted to $\mathrm{DC}$ by a rectifier but some harmonic signals are then generated from the rectifying diode. Therefore, a BPF or bandstop filter (BSF) with good performance is required to block these harmonic signals in the rectenna.

In this paper, a high order harmonic rejection microstrip BPF is introduced for a highly efficient rectenna design. The embedded BPF can suppress the $2^{\text {nd }}$ and $3^{\text {rd }}$ harmonics from the rectifying circuit, allowing the. proposed rectenna to achieve high conversion efficiency.

\section{II . Rectenna Design}

\section{2-1 Microstrip Antenna Design}

In general, a rectenna consists of an antenna, a bandpass filter (BPF), a rectifying diode, a low-pass filter (LPF), and a resistive or capacitive load.

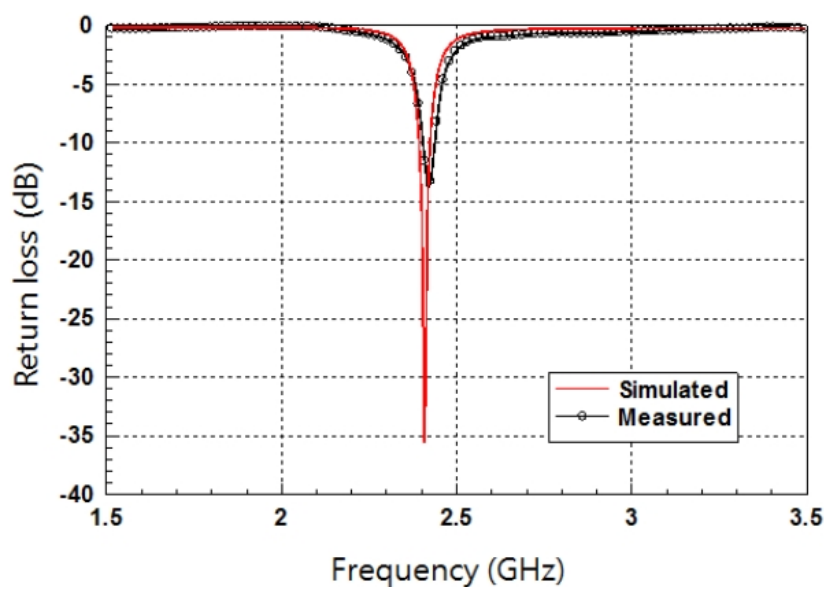

Fig. 1. Simulated and measured return losses of the microstrip patch antenna.

Manuscript received July 29, 2011 ; revised November 9, 2011. (ID No. 20110729-023J)

Electrical and Electronics Engineering, Chung-Ang University, Seoul, Republic of Korea.

Corresponding Author : Sungjoon Lim (e-mail : sungjoon@cau.ac.kr) 
We have designed a microstrip patch antenna to receive a RF signal at $2.4 \mathrm{GHz}$. The substrate is Rogers $\mathrm{RT} /$ Duroid 5880 with a thickness of $1.57 \mathrm{~mm}$ and a relative permittivity of 2.2 for all of our designs. The antenna has a length of $40.5 \mathrm{~mm}$ or $0.48 \lambda$ of square structure to resonate at $2.4 \mathrm{GHz}$. Its simulated and measured return losses are shown in Fig. 1. The simulated return loss is $-35 \mathrm{~dB}$ and the measured return loss is $-14 \mathrm{~dB}$.

\section{2-2 Harmonic Rejection BPF Design}

The microstrip BPF is designed by two microstrip ring resonators. The resonance frequency of BPF is defined by the length of (' $\mathrm{B}$ ' in Fig. 3(b)). It has $-18 \mathrm{~dB}$ return loss and a pass band at $2.4 \mathrm{GHz}$. The different path lengths (' $\mathrm{B}$ ' in Fig. 3(b)) of the ring resonators make transmission zero points at $1.5 \mathrm{GHz}$ and $3.2 \mathrm{GHz}$. The BPF has $2^{\text {nd }}$ and $3^{\text {rd }}$ harmonic rejection, which requires proper design of the gap size. To increase performance of insertion loss at harmonic frequencies, we have to modulate the length and width of the BPF. Harmonic rejection of BPF can block re-radiation signal energy to the antenna. The simulated and measured S-parameter results are shown in Fig. 2.

Simulated insertion losses are $-0.38,-7.9$, and -11.1 $\mathrm{dB}$ at 2.4, 4.8, and $7.2 \mathrm{GHz}$ and measured insertion losses are $-0.5,-8.4$, and $-9.7 \mathrm{~dB}$ at $2.4,4.8$, and 7.2 GHz. Therefore, we can expect that the proposed BPF will efficiently reject the harmonic signals generated by the nonlinear rectifying diode.

\section{2-3 Rectenna Design}

The proposed rectenna is illustrated with dimension details in Figs. 3(a), (b) and (c). It consists of the microstrip patch antenna, a BPF, matching stubs, a rectifying diode, an LPF, and a resistor. The two stubs are

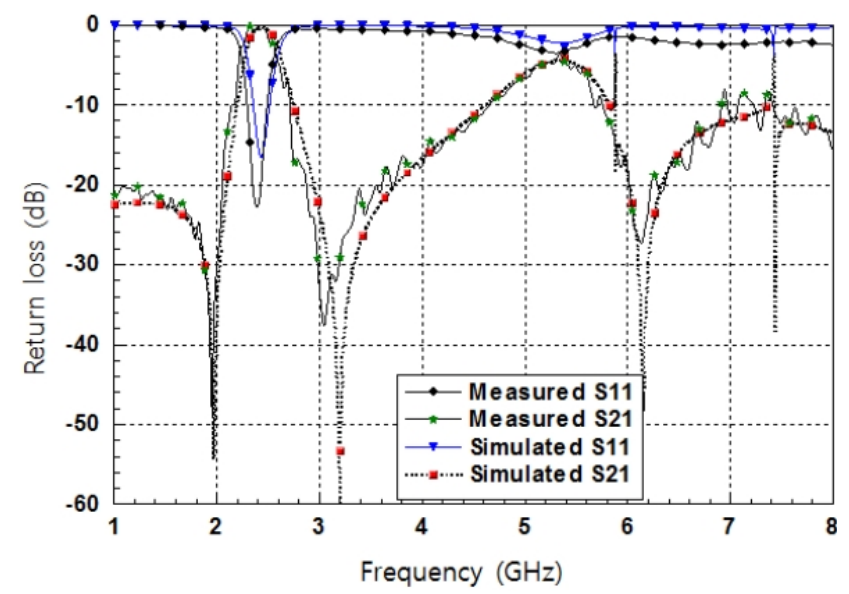

Fig. 2. Measured and simulated return losses of BPF.

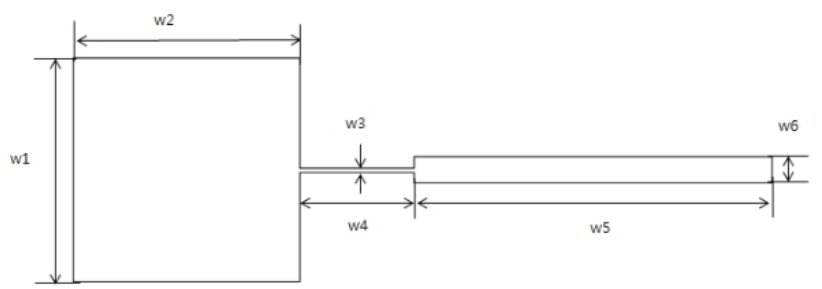

(a)

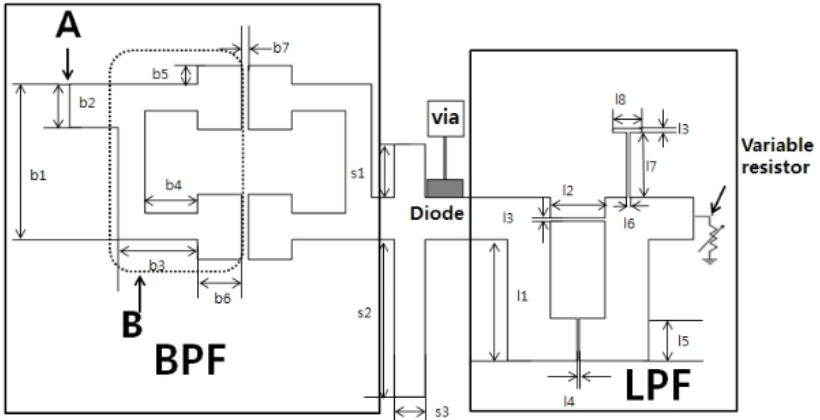

(b)

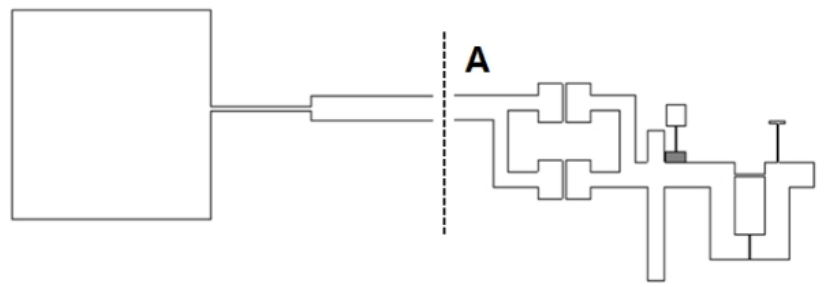

(c)

Fig. 3. (a) Layout of microstrip antenna. (b) Layout of rectifier. (c) Proposed rectenna.[dimensions: $w_{1}, w_{2}=$ $40.5, w_{3}=0.72, w_{4}=20.6, w_{5}=64.2, w_{6}=4.84, b_{1}=17.8$, $b_{2}=4.84, b_{3}=9, \quad b_{4}=6, \quad b_{5}=2.2, \quad b_{6}=5, \quad b_{7}=0.8, s_{1}=61$, $s_{2}=18, s_{3}=3, l_{1}=13.9, l_{2}=6.2, l_{3}, l_{4}, l_{6}=0.2, l_{5}=4.84, l_{7}=$ $7.35, l_{8}=3.25, l_{9}=0.5$ (units $\left.\left.=\mathrm{mm}\right)\right]$.

inserted between the BPF and the rectifying diode. In Fig. 3(b), (A) is the input of the rectifier. A diode is connected to the ground plane by a via. A variable load resistance is located between the LPF and the ground plane.

We used a Schottky barrier diode (HSMS 2820) for converting RF to DC. The diode is connected in parallel with the microstrip line and ground plane. Because of the diode's non-linearity, several harmonic signals are generated and re-radiated through the antenna. In order to block these signals, the harmonic rejection BPF is designed between the antenna and the diode.

Finally, the LPF can pass the converted DC signal and reject the fundamental and harmonic RF signals. The conversion efficiency is varied by the resistance value at the load. The optimum resistance is decided by the variable resistor.

\section{Rectenna Performance}




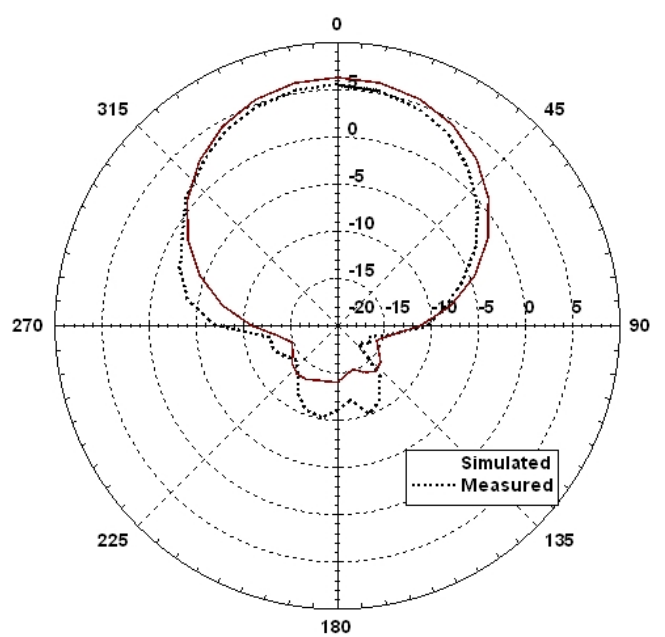

Fig. 4. Simulated and measured radiation patterns of microstrip antenna.

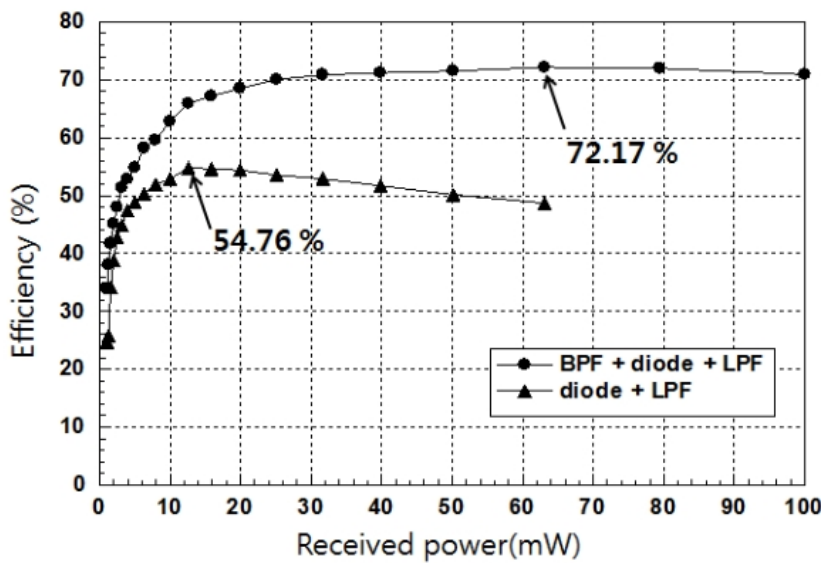

Fig. 5. Conversion efficiency versus received power.

Before measuring the rectenna performances, the antenna's radiation pattern is first measured. Fig. 4 shows the simulated and measured radiation patterns at 2.4 GHz. The microstrip antenna has $5.4 \mathrm{dBi}$ of peak gain at $2.4 \mathrm{GHz}$.

First, the performance of the rectifier is measured by removing the antenna. Fig. 5 plots the measured efficiency versus the received RF power for two cases: (BPF, diode, and LPF) and (diode and LPF). The conversion efficiency $(\eta)$ is defined by:

$$
\eta[\%]=\frac{P_{D C}}{P_{R F}} \times 100
$$

where $P_{D C}$ and $P_{R F}$ are the output DC power at the load and the input RF power, respectively. In this test setup, $P_{R F}$ is the transmitted RF power at the rectifier. The measured maximum conversion efficiency is $72.17 \%$ when the input power is $63.09 \mathrm{~mW}$ at $2.4 \mathrm{GHz}$ and the load resistance is $932 \Omega$. The other case without BPF has a

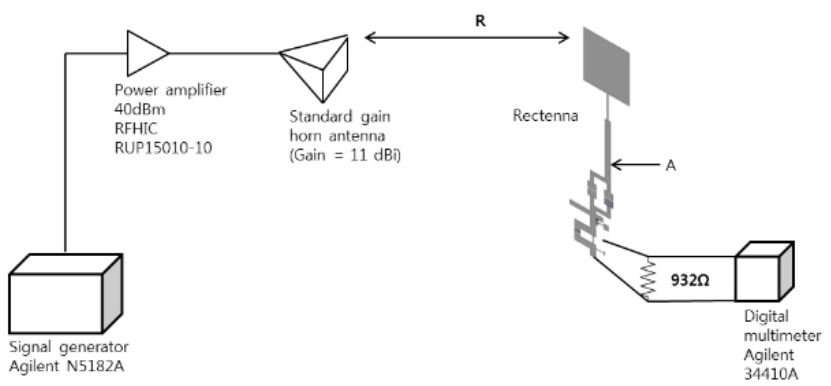

Fig. 6. Rectenna measurement setup.

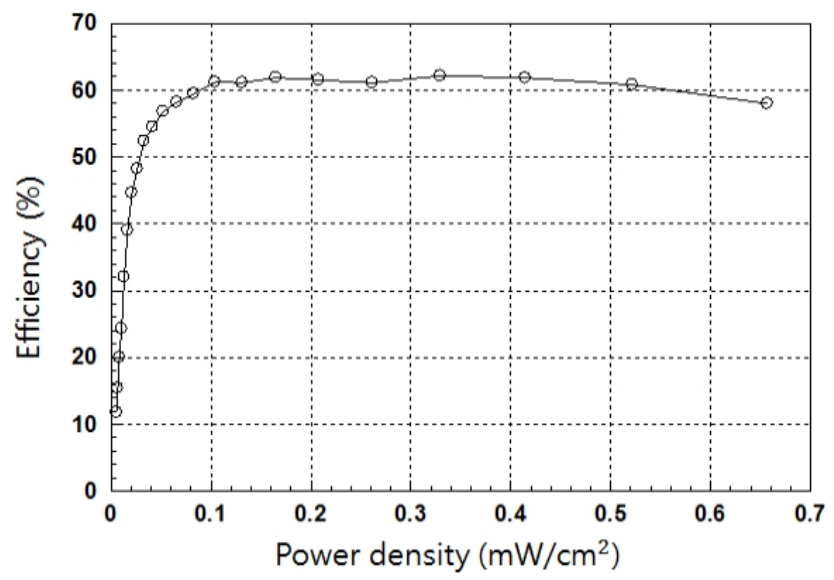

Fig. 7. Conversion efficiency versus power density.

conversion efficiency of $54.76 \%$ at $12.58 \mathrm{~mW}$. This implies that the BPF successfully reduces re-radiation signals. Consequently, the conversion efficiency is increased more than $17 \%$ by harmonic rejection $\mathrm{BPF}$.

Next, the rectenna performance is measured in free space. Its measurement setup is illustrated in Fig. 6. A $2.4 \mathrm{GHz}$ continuous wave $(\mathrm{CW})$ signal is amplified and transmitted through a standard gain horn antenna. The distance between the transmitting horn antenna and the proposed rectenna is $29 \mathrm{~cm}$. Fig. 7 shows the measured efficiency versus the power density at $2.4 \mathrm{GHz}$. The load resistance is $932 \Omega$.

When the transmitting RF power from the power amplifier is $P_{t}$ and the horn antenna's gain is $G_{t}$, the received RF power $\left(P_{r}\right)$ is calculated by the Friis equation.

$$
P_{r}=\frac{G_{t} G_{r} \lambda^{2}}{(4 \pi R)^{2}} P_{t},
$$

where $G_{r}, \lambda$, and $R$ are the receiving patch antenna's gain and wavelength, and the distance between the transmitting antenna and the rectenna, respectively.

Since $A_{e}$ represents the effective area defined by:

$$
A_{e}=\frac{G_{r} \lambda^{2}}{4 \pi}
$$


the received power can be expressed as: .

$$
P_{r}=\frac{G_{t} P_{t} A_{e}}{4 \pi R^{2}},
$$

Since the power density $\left(P_{D}\right)$ is defined by

$$
P_{D}=\frac{P_{r}}{A_{e}},
$$

the conversion efficiency can be modified as:

$$
\eta[\%]=\frac{P_{D C}}{A_{e} P_{D}} \times 100 .
$$

By changing the transmitted power from the signal generator, the $\mathrm{DC}$ power at the rectenna is measured and the efficiency is calculated by Eq. (2) (6). We use the value $11 \mathrm{dBi}$ for $G_{t}, 5.4$ and $10 \mathrm{dBi}$ for $G_{r}, 12.5 \mathrm{~cm}$ for $\lambda_{0}^{2}$ and $29 \mathrm{~cm}$ of $\mathrm{R}$ in the present measurement setup. Fig. 7 shows that the maximum conversion efficiency is $62.13 \%$.

\section{IV . Conclusion}

A highly efficient rectenna is proposed at $2.4 \mathrm{GHz}$. The high conversion efficiency is achieved by a harmonic rejection BPF. The BPF is experimentally confirmed to provide a successful suppression of the $2^{\text {nd }}$ and $3^{\text {rd }}$ harmonic signals. When this BPF is employed in a rectenna, $72.17 \%$ of the maximum conversion efficiency is achieved at $63.09 \mathrm{~mW}$. Experimental results show that the conversion efficiency improves more than $17 \%$ by using harmonic rejection BPF.

This research was supported by Basic Science Research Program through the National Research Foundation of Korea (NRF) funded by the Ministry of Education, Science and Technology (2010-0004315).

\section{References}

[1] Y. H. Suh, K. Chang, "A high efficiency dual-frequency rectenna for 2.45- and 5.8- GHz wireless power transmission," IEEE Trans. Microwave Theory Tech., vol. 50, no. 7, pp. 1784-1789, Jul. 2002.

[2] J. Y. Park, S. M. Han, and T. Itoh, "A rectenna design with harmonic-rejecting circular-sector antenna," IEEE Antennas and Wireless Propagation Letter, vol. 3, pp. 52-54, 2004.

[3] J. A. Hagerty, F. B. Helmbrecht, W. H. McCalpin, R. Zane, and Z. B. Popovic, "Recycling ambient microwave energy with broad-band rectenna arrays," IEEE Trans. Microwave Theory Tech., vol. 52, pp. 10141024, Mar. 2004.

[4] Y. J. Ren, K. Chang, "5.8-GHz circularly polarized dual-diode rectenna and rectenna array for microwave power transmission," IEEE Trans. Microwave Theory Tech., vol. 54, no. 4, pp. 1495-1502, Apr. 2006.

[5] S. K. Parui, S. Das, "Performance enhancement of microstrip open loop resonator band pass filter by defected ground structures," Proc. IEEE Workshop Antenna Technol. Small Smart Antennas Metamater, pp. 483-486, 2007.

[6] B. Strassner, K. Chang, "5.8-GHz circularly polarized rectifying antenna for wireless microwave power transmission," IEEE Trans. Microwave Theory Tech., vol. 50, pp. 1870-1876, Aug. 2002.

[7] T. Paing, J. Shin, R. Zane, and Z. Popovic, "Resistor emulation approach to low-power RF energy harvesting," IEEE trans. Power Electronics., vol. 23, pp 1494-1501, May. 2008.

[8] D. M. Pozar, Microwave Engineering, Wiley, 3rd Ed., pp. 647-648, 2005. 


\section{Younghwan Kim}

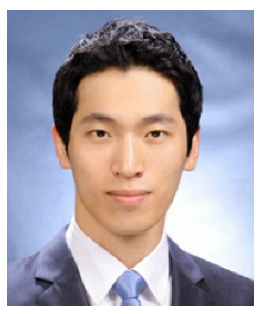

received the B.S. degree in electrical and electronics engineering from Chung-Ang University in 2011, Seoul, Republic of Korea. $\mathrm{He}$ is currently working toward a M.S. degree in electrical and electronics engineering at Chung Ang University. His research interests include antennas and wireless power transmission.

\section{Sungjoon Lim}

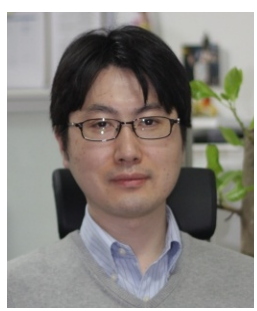

received the B.S. degree in electronic engineering from Yonsei University, Seoul, Korea, in 2002, and the M.S. and Ph.D. degrees in electrical engineering from the University of California at Los Angeles (UCLA), in 2004 and 2006, respectively. After a postdoctoral position at the Integrated Nanosystem Research Facility (INRF), the University of California at Irvine, he joined the School of Electrical and Electronics Engineering, Chung-Ang University, Seoul, Korea, in 2007, where he is currently an Assistant Professor. He has authored and coauthored more than 60 technical conference, letter and journal papers. His research interests include engineered metamaterial structures, printed antennas, arrays, and RF MEMS applications. He is also interested in wireless power transmission.

Dr. Lim received the Institution of Engineering and Technology (IET) Premium Award in 2009. 